\title{
Inflammatory myofibroblastic tumor of the prostate after transurethral resection of the prostate with negative expression of anaplastic lymphoma kinase: a case report
}

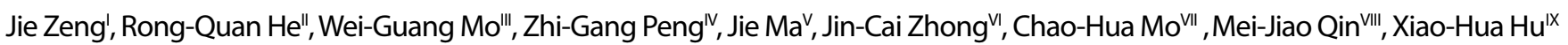

'MD, MSc. Postgraduate Student, Department of Medical Oncology, First Affiliated Hospital of Guangxi Medical University, Nanning, Guangxi, China.

(Dorcid.org/0000-0002-8582-7319

"MD. Postgraduate Student, Department of Medical Oncology, First Affiliated Hospital of Guangxi Medical University, Nanning, Guangxi, China.

(Dorcid.org/0000-0002-7752-2080

"'MSc. Technician, Department of Pathology, First Affiliated Hospital of Guangxi Medical University, Nanning, Guangxi, China.

(Dorcid.org/0000-0001-7635-6984

"MD, MSc. Professor, Department of Medical Oncology, First Affiliated Hospital of Guangxi Medical University, Nanning, Guangxi, China. (D)orcid.org/0000-0003-4691-1902

'MD, MSc. Professor, Department of Medical Oncology, First Affiliated Hospital of Guangxi Medical University, Nanning, Guangxi, China.

(Dorcid.org/0000-0002-8745-8772

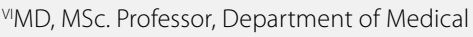
Oncology, First Affiliated Hospital of Guangxi Medical University, Nanning, Guangxi, China.

Dorcid.org/0000-0001-8293-180X

"IIMD, MSc. Attending Physician and

Postgraduate Student, Department of

Pathology, First Affiliated Hospital of Guangxi Medical University, Nanning, Guangxi, China.

(D)orcid.org/0000-0002-0884-3905

VIIIMD, MSc. Postgraduate Student, Department of Medical Oncology, First Affiliated Hospital of Guangxi Medical University, Nanning,

Guangxi, China.

(D)orcid.org/0000-0002-5939-1654

${ }^{1 \times} M D, M S c$. Professor, Department of Medical Oncology, First Affiliated Hospital of Guangxi Medical University, Nanning, Guangxi, China. (Dorcid.org/0000-0002-2161-1324

KEY WORDS:

Granuloma, plasma cell.

Inflammation.

Prostate.

\begin{abstract}
CONTEXT: Inflammatory myofibroblastic tumors are a rare type of soft-tissue tumor. Inflammatory myofibroblastic tumors are characterized by rearrangements involving the anaplastic lymphoma kinase gene locus on 2 p23.

CASE REPORT:We report the case of a 67 -year-old Chinese male who presented with dysuria and fever. Magnetic resonance imaging showed an irregular prostatic mass with an isointense signal and obscure boundary. Histopathological evaluation showed that the mass consisted mainly of spindle-shaped cells. Immunohistochemical evaluation showed that the tumor cells were negative for anaplastic lymphoma kinase.

CONCLUSIONS: Inflammatory myofibroblastic prostate tumors are rare lesions with unclear etiology. The pathological diagnosis is very important.
\end{abstract}

\section{INTRODUCTION}

Inflammatory myofibroblastic tumors can be found in various parts of the body and are frequently identified in the lung or abdominal cavity of children and young adults. Inflammatory myofibroblastic tumors of the urinary tract present more often in kidneys. Prostatic inflammatory myofibroblastic tumors are extremely rare. Since the first case in 2012, only one further case of prostatic inflammatory myofibroblastic tumor has been reported in the literature. ${ }^{1,2}$ The usual clinical presentation of inflammatory myofibroblastic prostate tumors consists of dysuria and acute urinary retention. Differentiation of inflammatory myofibroblastic prostate tumors from malignant prostate tumors through imaging and laboratory tests is difficult. A case of prostatic inflammatory myofibroblastic tumor observed after transurethral resection of the prostate to treat prostate hyperplasia in a 67 -year-old man is presented in this report.

\section{CASE REPORT}

A 67-year-old Chinese male presented with dysuria and fever for a month. He had a history of transurethral resection of the prostate to treat prostate hyperplasia four years earlier. Laboratory tests such as blood count and urinalysis were within the normal range. His serum total prostate-specific antigen level was $8.49 \mathrm{ng} / \mathrm{ml}$ (normal reference range: 0.00-4.00 ng/ml) and his free prostate-specific antigen level was $1.42 \mathrm{ng} / \mathrm{ml}$ (normal reference range: $0.00-0.93 \mathrm{ng} / \mathrm{ml}$ ).

Subsequently, multiple echoless masses and calcification were found using ultrasonography. Magnetic resonance imaging then demonstrated an irregular prostatic mass with an obscure boundary and isointense signal on T1 and T2-weighted images (Figure 1A and Figure 1B). The lesion showed obvious enhancement in the early phase, on a contrast-enhanced magnetic resonance imaging scan (Figure 2). Multiple enlarged lymph nodes were found in the right iliac vascular area and in the inguinal area bilaterally.

A transperineal biopsy of the prostate was performed on this lesion because the diagnosis was still unclear. Histopathological evaluation showed that the mass consisted mainly of spindle-shaped cells and a chronic inflammatory component consisting of plasma cells (Figure 3A). Immunohistochemical evaluation showed that the tumor cells were positive for myoepithelial markers, including desmin, calponin, vimentin, actin and smooth muscle actin (Figure 3B-E), but that they were negative for CD117, CD10, S100 and anaplastic lymphoma kinase (Figure 3F). 
Based on the histological and immunohistochemical findings, a pathological diagnosis of inflammatory myofibroblastic tumor was made. No further specific treatment was provided. The patient was followed up for two years and no evidence of recurrence or metastasis was noted.

\section{DISCUSSION}

Inflammatory myofibroblastic tumors were once considered to be inflammatory pseudotumors, xanthogranulomas, plasma-cell granulomas or plasma-cell pseudotumors. ${ }^{3}$ However, inflammatory myofibroblastic tumors and inflammatory pseudotumors are
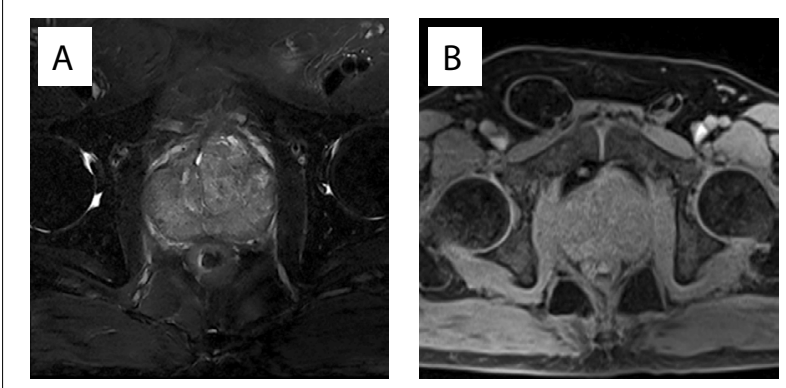

Figure 1. Irregular prostatic mass showing an isointense signal on T1-weighted images (A) and T2-weighted images (B), with an obscure boundary. completely different pathological concepts with different pathological manifestations. Inflammatory pseudotumors are characterized by an inflammatory infiltrate consisting of lymphocytes,

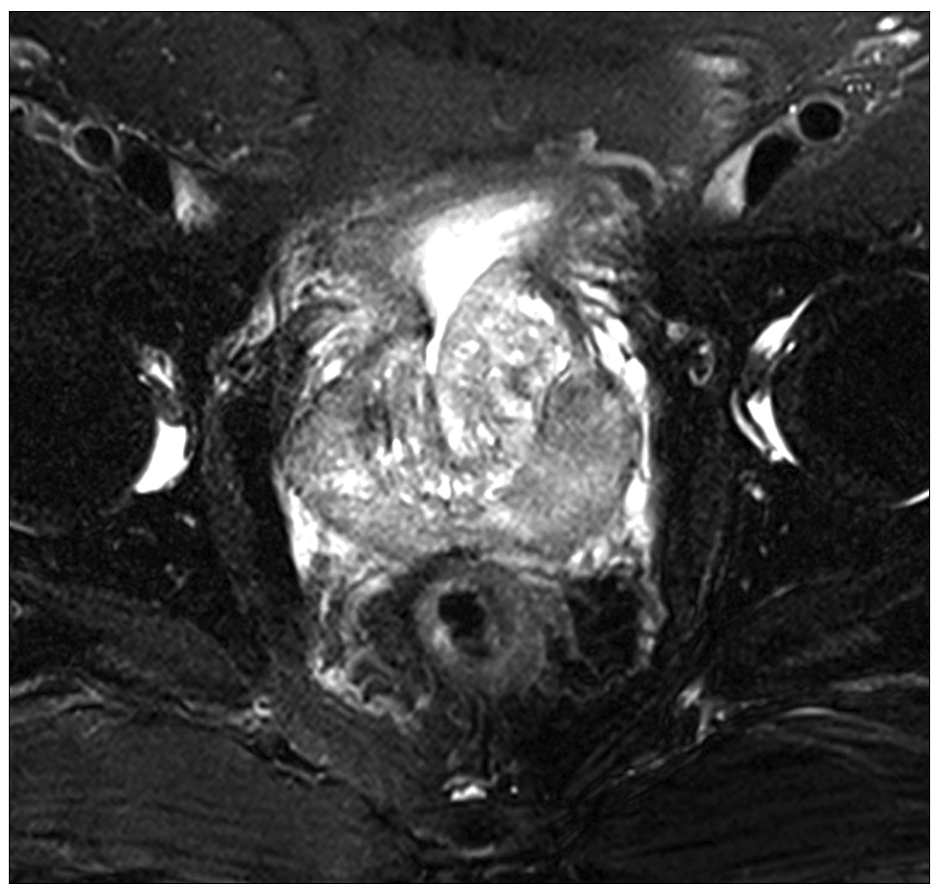

Figure 2. Contrast-enhanced magnetic resonance imaging showing clear enhancement in the early phase of the lesion.

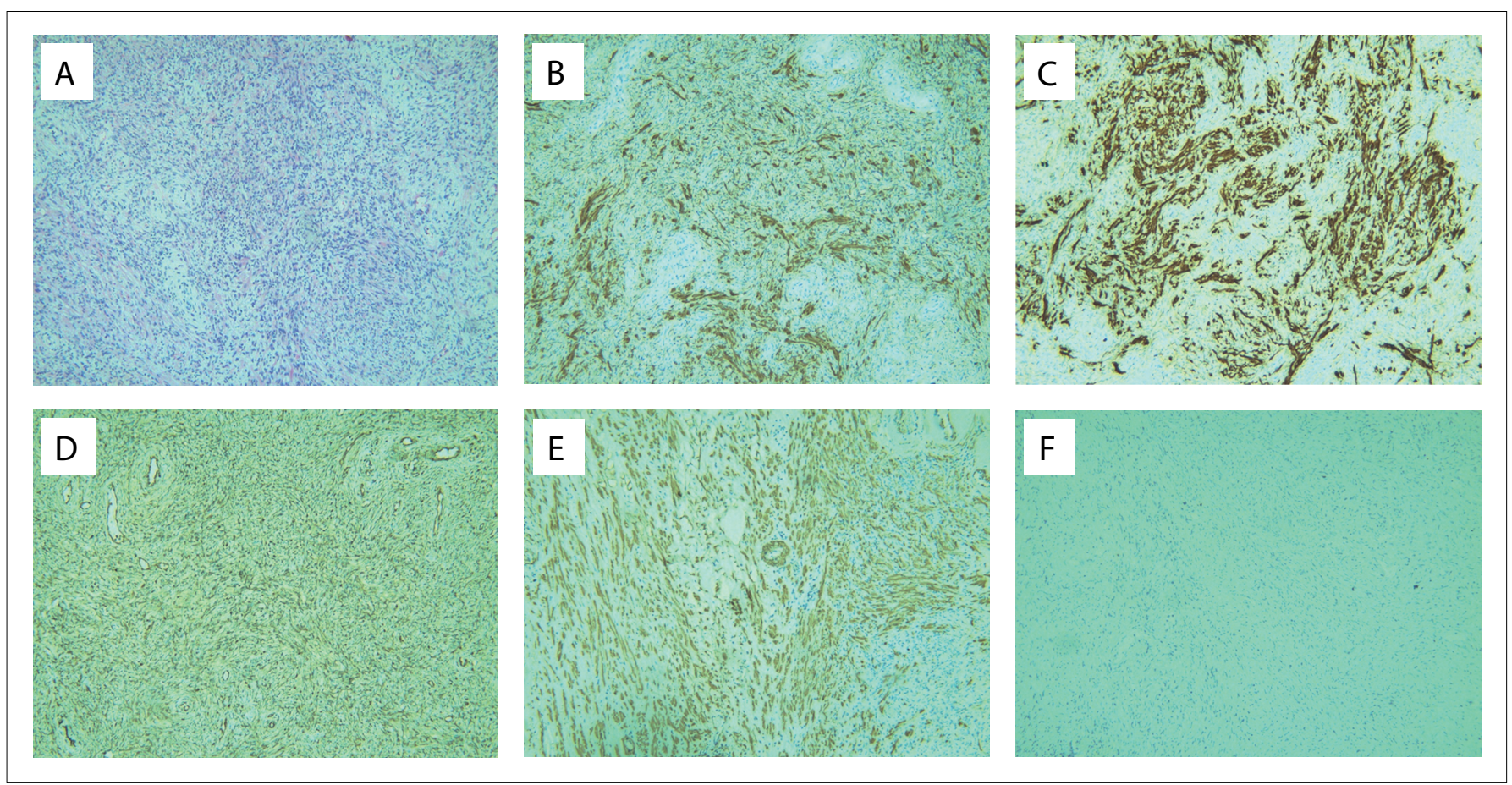

Figure 3. Histopathological and immunohistochemical sections through a prostatic inflammatory myofibroblastic tumor. The tumor consisted mainly of spindle-shaped cells and a chronic inflammatory component consisting of plasma cells (A); hematoxylin-eosin staining ( $\times 100)$. Immunohistochemical sections showed that the tumor cells were positive for desmin (B), calponin (C), vimentin (D) and smooth muscle actin (SMA) (E), but were negative for anaplastic lymphoma kinase (ALK) (F). 
plasma cells and histiocytes admixed with variable proportions of fibroblasts and myofibroblasts. On the other hand, inflammatory myofibroblastic tumors were classified as soft-tissue tumors by the World Health Organization in 2002, and consist mainly of differentiated myofibroblastic spindle cells, usually accompanied by numerous plasma cells, eosinophilic granulocytes and/or lymphocytes. Inflammatory myofibroblastic tumors are characterized by rearrangements involving the anaplastic lymphoma kinase gene locus on $2 \mathrm{p} 23 .^{4}$

The etiology of inflammatory myofibroblastic tumors remains unknown. ${ }^{5}$ Inflammatory myofibroblastic tumors are frequently associated with inflammation, surgery and trauma. Therefore, inflammatory myofibroblastic tumors may be the body's response to injury caused by hyperplasia.

The neoplastic processes of inflammatory myofibroblastic tumors are characterized by gene rearrangement or fusion. Next-generation sequencing revealed that $85 \%$ of the cases evaluated harbored kinase fusions involving anaplastic lymphoma kinase, ROS1 or PDGF $\beta$. The fusion partners identified thus far include the TPM3/4, CLTC and RANBP2 genes. A subset of anaplastic lymphoma kinase-negative inflammatory myofibroblastic tumors demonstrated ROS-1 gene fusions. ${ }^{4,6}$

Inflammatory myofibroblastic tumors can be found primarily in children and young adults, and they rarely occur in patients over 40 years old. ${ }^{7}$ Although inflammatory myofibroblastic tumors are more commonly recognized in the lungs, they can also be observed in several other sites including the breasts, liver, spleen, thyroid, pancreas, urinary tract, peritoneum, retroperitoneum, lymph nodes, gastrointestinal tract and central nervous system. ${ }^{8}$ Inflammatory myofibroblastic tumors of the urinary tract have been reported more often in the kidneys.

Prostatic inflammatory myofibroblastic tumors are extremely rare. Since the first case in 2012, only one further case of inflammatory myofibroblastic prostate tumor has been reported (Table 1). ${ }^{1,2}$ In the present case, we reported that an inflammatory myofibroblastic tumor was found in an elderly man after transurethral resection of the prostate to treat prostate hyperplasia. This case was negative for anaplastic lymphoma kinase.

The final diagnosis of an inflammatory myofibroblastic tumor cannot be made from the features of the clinical manifestation, laboratory tests or radiological examination. The clinical manifestations of inflammatory myofibroblastic tumors are diverse in different sites. Urinary inflammatory myofibroblastic tumors present with obstructive symptoms such as dysuria, urinary frequency and urinary retention. Rectal examination may reveal a palpable mass. Inflammatory myofibroblastic tumors usually demonstrate low signal intensity on both T1 and T2-weighted images and show obvious enhancement on contrast-enhanced magnetic resonance imaging scans. ${ }^{9}$
However, these features are not distinctly different from those of prostate hyperplasia and prostate cancer. Therefore, the pathological diagnosis is especially important. These tumors are infiltrated by plasma cells, lymphocytic plasma cells, eosinophils and other inflammatory cells. Immunohistochemically, the spindle cells are positive for expression of muscle-derived proteins. Vimentin is often expressed diffusely, whereas smooth muscle actin and desmin are expressed focally or diffusely. ${ }^{10}$

This patient showed symptoms of dysuria, with abnormal prostate-specific antigen levels. A definite diagnosis cannot be made from symptoms, laboratory testing or imaging examinations. Ultimately, the diagnosis of an inflammatory myofibroblastic tumor was made using histological and immunohistochemical evaluations.

A variety of treatment methods for inflammatory myofibroblastic tumors have been reported, including chemotherapy, radiation therapy, non-steroidal anti-inflammatory drugs and glucocorticoid therapy. Additionally, there is no exact index to predict the risk of recurrence and metastasis.

Responses to the tyrosine kinase inhibitor crizotinib have been documented in patients with anaplastic lymphoma kinase-positive inflammatory myofibroblastic tumors. However, a new study by Lovly et al. found that this also had clear curative effects in patients with ROS 1 fusion. ${ }^{6}$ For extrapulmonary inflammatory myofibroblastic tumors, surgical excision is still preferred. ${ }^{11,12}$ Coffin et al.

Table 1. Search of the literature in medical databases for inflammatory myofibroblastic tumor of the prostate. The literature search was conducted on July 28, 2017

\begin{tabular}{|c|c|c|c|}
\hline Database & Search strategies & $\begin{array}{l}\text { Papers } \\
\text { found }\end{array}$ & $\begin{array}{c}\text { Related } \\
\text { papers }\end{array}$ \\
\hline $\begin{array}{l}\text { MEDLINE } \\
\text { (via PubMed) }\end{array}$ & $\begin{array}{l}\text { \#1 (“Granuloma, Plasma } \\
\text { Cell"[Mesh]) } \\
\text { \#2 (Prostate"[Mesh]) } \\
\text { \#3 (“Transurethral Resection of } \\
\text { Prostate"[Mesh]) } \\
\text { \#4 \#2 OR \#3 } \\
\text { \#5 \#1 AND \#4 }\end{array}$ & 3 & 2 \\
\hline $\begin{array}{l}\text { Embase } \\
\text { (via Elsevier) }\end{array}$ & $\begin{array}{l}\text { \#1 (inflammatory } \\
\text { myofibroblastic tumor) OR } \\
\text { (inflammatory myofibroblastic } \\
\text { tumour) OR (Granuloma Plasma } \\
\text { Cell) } \\
\text { \#2 Prostate OR (Transurethral } \\
\text { Resection of Prostate) } \\
\text { \#3 \#1 AND \#2 }\end{array}$ & 17 & 2 \\
\hline $\begin{array}{l}\text { LILACS } \\
\text { (via BVS) }\end{array}$ & $\begin{array}{l}\text { \#1 mh:(Granuloma, Plasma Cell) } \\
\text { \#2 mh:(Prostate) } \\
\text { \#3 mh:(Transurethral Resection } \\
\text { of Prostate) } \\
\text { \#4 \#2 OR \#3 } \\
\text { \#5 \#1 AND \#4 }\end{array}$ & 2 & 1 \\
\hline
\end{tabular}

BVS = Biblioteca Virtual em Saúde; LILACS = Literatura Latino Americana e do Caribe em Ciências da Saúde. 
reported that negative expression of anaplastic lymphoma kinase correlated with a risk of recurrence and metastasis. ${ }^{6,13,14}$ However, a review of extrapulmonary inflammatory myofibroblastic tumors documented a recurrence rate of $31 \%$ among anaplastic lymphoma kinase-negative tumors and 69\% among anaplastic lymphoma kinase-positive tumors. Anaplastic lymphoma kinase gene rearrangements occurred in approximately $50-75 \%$ of cases of inflammatory myofibroblastic tumors. ${ }^{7,15}$

Neither of the previous reports of prostatic inflammatory myofibroblastic tumors measured anaplastic lymphoma kinase levels. One of these patients suffered from recurrence and distant metastasis and subsequently died., ${ }^{1,2}$

The patient of our report did not express anaplastic lymphoma kinase. No further specific treatment was provided. The patient was followed up for two years and no evidence of recurrence or metastasis was noted. This suggests that treatment approaches for inflammatory myofibroblastic tumor patients can vary and surgery may not be essential.

\section{CONCLUSION}

In conclusion, prostatic inflammatory myofibroblastic tumors are rare lesions with unclear etiology. A definitive diagnosis cannot be made from symptoms, laboratory testing and imaging studies. The pathological diagnosis is very important. Regarding treatment methods, although surgery is not essential for treating prostatic inflammatory myofibroblastic tumors that are negative for anaplastic lymphoma kinase expression, long-term follow-up is necessary.

\section{REFERENCES}

1. Zhang HH, Qi F, Zu XB, et al. Recurrence of inflammatory myofibroblastic tumor in bladder secondary to prostate treated with laparoscopic radical cystectomy. Med Sci Monit. 2012;18(8):CS63-66. PMID: 22847204.

2. Liu C, Zhao X, Zhao Z, et al. Malignant inflammatory myofibroblastic tumor of the prostate. J Clin Oncol. 2013;31(10):e144-7. PMID: 23401456.

3. Zhao HD, WU T, Wang JQ, et al. Primary inflammatory myofibroblastic tumor of the breast with rapid recurrence and metastasis: A case report. Oncol Lett. 2013;5(1):97-100. PMID: 23255901.

4. SurabhiVR, Chua S, Patel RP, et al. Inflammatory Myofibroblastic Tumors: Current Update. Radiol Clin North Am. 2016;54(3):553-63. PMID: 27153788.

5. González MG, Vela D, Álvarez M, Caramés J. Inflammatory myofibroblastic duodenal tumor: A rare cause of massive intestinal bleeding. Cancer Biomark. 2016;16(4):555-7. PMID: 27002758.

6. Lovly CM, Gupta A, Lipson D, et al. Inflammatory myofibroblastic tumors harbor multiple potentially actionable kinase fusions. Cancer Discov. 2014;4(8):889-95. PMID: 24875859
7. Zhou Y, Zhu J, Zhang Y, Jiang J, Jia M. An inflammatory myofibroblastic tumour of the breast with ALK overexpression. BMJ Case Rep. 2013. pii: bcr0720114474. PMID: 23386486.

8. Khanafshar E, Phillipson J, Schammel DP, et al. Inflammatory myofibroblastic tumor of the breast. Ann Diagn Pathol. 2005;9(3):123-9. PMID: 15944952

9. Takayama Y, Yabuuchi H, Matsuo Y, et al. Computed tomographic and magnetic resonance features of inflammatory myofibroblastic tumor of the lung in children. Radiat Med. 2008;26(10):613-7. PMID: 19132493

10. Gao F, Zhong R, Li GH, Zhang WD. Computed tomography and magnetic resonance imaging findings of inflammatory myofibroblastic tumors of the head and neck. Acta Radiol. 2014;55(4):434-40. PMID: 23966365.

11. Germanidis G, Xanthakis I, Tsitouridis I, et al. Regression of inflammatory myofibroblastic tumor of the gastrointestinal tract under infliximab treatment. Dig Dis Sci. 2005;5(2):262-5. PMID: 15745083.

12. Li HB, Xu YM, Yu JJ. Diagnostic puzzle of inflammatory pseudotumor of the urinary bladder: a case report with brief literature review. South Med J. 2010;103(6):563-6. PMID: 20710142.

13. Coffin $C M$, Hornick JL, Fletcher CD. Inflammatory myofibroblastic tumor: comparison of clinicopathologic, histologic, and immunohistochemical features including ALK expression in atypical and aggressive cases. Am J Surg Pathol. 2007;31(4):509-20. PMID: 17414097

14. Teoh JY, Chan NH, Cheung HY, Hou SS, Ng CF. Inflammatory myofibroblastic tumors of the urinary bladder: a systematic review. Urology. 2014;84(3):503-8. PMID: 25168523.

15. Satomi T, Watanabe M, Matsubayashi J, Nagao T, Chiba H. A successfully treated inflammatory myofibroblastic tumor of the mandible with long-term follow-up and review of the literature. Med Mol Morphol. 2010;43(3):185-91. PMID: 20857269.

Conflict of interest: None

Sources of interest: None

Date of first submission: March 16, 2017

Last received: March 16, 2017

Accepted: April 7, 2017

\section{Address for correspondence:}

Xiao-Hua Hu

Department of Medical Oncology

First Affiliated Hospital of Guangxi Medical University

6 Shuangyong Road

Nanning - Guangxi — China

Tel. 008613507719316

E-mail: gxmuhxh@163.com 